Check for updates

Cite this: Chem. Sci., 2019, 10, 7352 have been paid for by the Royal Society of Chemistry
๑ All publication charges for this article

\section{Two-photon-excited ultralong organic room temperature phosphorescence by dual-channel triplet harvesting $\dagger$}

\author{
Zhu Mao, (ID $\ddagger^{a}$ Zhan Yang,,$^{\mathrm{a}}$ Chao Xu, $\dot{+}^{\mathrm{b}}$ Zongliang Xie, ${ }^{\mathrm{a}}$ Long Jiang, ${ }^{\mathrm{a}}$ \\ Feng Long Gu, (D) ${ }^{\mathrm{b}}$ Juan Zhao, (D) *a Yi Zhang, (D) a Matthew P. Aldred ${ }^{\mathrm{a}}$ \\ and Zhenguo $\mathrm{Chi}\left(\mathbb{D}^{\text {a }}\right.$
}

\begin{abstract}
Due to inefficient molecular design strategies, two-photon-excited ultralong organic room temperature phosphorescence (TPUOP) has not yet been reported in single-component materials. Herein, we present an innovative design method by dual-channel triplet harvesting to obtain the first bright TPUOP molecule with a lifetime of $0.84 \mathrm{~s}$ and a quantum efficiency of $16.6 \%$. In compound $\mathrm{o}-\mathrm{Cz}$ the donor and acceptor units are connected at the ortho position of benzophenone, showing intramolecular space charge transfer. Therefore, the two-photon absorption ability is improved due to the enhanced charge transfer character. Moreover, the small energy gap boosts dual-channel triplet harvesting via ultralong thermally activated delayed fluorescence and $\mathrm{H}$-aggregation phosphorescence, which suppresses the long-lived triplet concentration quenching. Through two-photon absorption, a near-infrared laser ( 808 $\mathrm{nm}$ ) is able to trigger the obvious ultralong emission under ambient conditions. This research work provides valuable guidance for designing near-infrared-excited ultralong organic room temperature phosphorescence materials.
\end{abstract}

Received 10th May 2019

Accepted 20th June 2019

DOI: $10.1039 / c 9 s c 02282 a$

rsc.li/chemical-science

\section{Introduction}

Ultralong organic room temperature phosphorescence (UOP) materials, in which the light generated can last from several seconds to hours after removal of the excitation source, have been attracting considerable research interest. These types of materials are important for certain practical applications such as in emergency lighting, anti-counterfeiting, sensors, and biological sciences. ${ }^{1-5}$ Recently, strategies such as $\mathrm{H}$ aggregation, crystallization, guest-host doping, intermolecular electronic coupling, and molecular self-assembly have all been adopted in order to promote the development of UOP materials. ${ }^{6-13}$ However, the reported UOP materials are only triggered by a single-photon of ultraviolet or blue light, ${ }^{14,15}$ restricting their actual utilization. Alternatively, two-photon absorption (TPA) utilizes the near-infrared (NIR) wavelengths that are more

${ }^{a}$ PCFM Lab, GDHPPC Lab, Guangdong Engineering Technology, Research Center for High-performance Organic and Polymer Photo-electric, Functional Films, State Key Laboratory of OEMT, School of Chemistry, Sun Yat-Sen University, Guangzhou 510275, PR China.E-mail: zhaoj95@mail.sysu.edu.cn

${ }^{b}$ Key Laboratory of Theoretical Chemistry of Environment, Ministry of Education, School of Chemistry \& Environment, South China Normal University, Guangzhou 510006, PR China

$\dagger$ Electronic supplementary information (ESI) available. CCDC 1885292. For ESI and crystallographic data in CIF or other electronic format see DOI: 10.1039/c9sc02282a

\$ Dr Z. Mao, Dr Z. Yang and Dr C. Xu contributed equally to this work. accessible for biological sciences due to higher spatial resolution, less phototoxicity and deeper tissue penetration..$^{16,17}$ Although progress has been made, systems exhibiting twophoton-induced ultralong emission are limited to inorganic and organic-inorganic hybrid materials, ${ }^{18-21}$ and two-photonexcited ultralong organic room temperature phosphorescence (TPUOP) materials from purely organic single component materials are unfortunately not yet discovered. The design and synthesis of TPUOP materials still remains a challenge because of the lack of efficient molecular design strategies.

There are several aspects that need to be considered for the design of efficient TPUOP emitters. It is desirable for the material to possess the following: (i) a longer wavelength absorption so that excitation can be maintained in the NIR spectral region for efficient tissue penetration, (ii) a high phosphorescence efficiency $\left(\Phi_{\mathrm{T}}\right)$ in order to lower the excitation incident energy, and (iii) a long phosphorescence lifetime to avoid continuous excitation. For high $\Phi_{\mathrm{T}}$ one should suppress the non-radiative pathways and enlarge the charge delocalization for red-shifted absorption. Hence, recent research studies have focused on enhancing intermolecular interactions to suppress non-radiative pathways, and indeed have successfully achieved high $\Phi_{\mathrm{T}}$ and a long lifetime. ${ }^{5-15}$ However, less attention has been paid to the intramolecular steric hindrance effects in reducing the excited-state vibrations and regulating the charge transfer characteristics of ultralong phosphorescent compounds. 


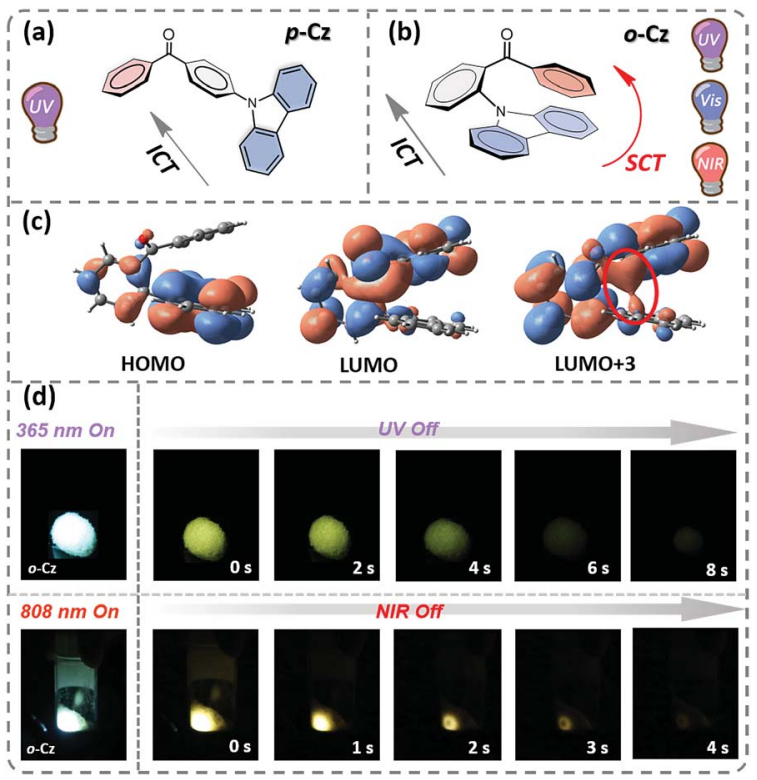

Fig. 1 (a) Chemical structure of $p-C z$, the grey arrow represents through-bond intramolecular charge transfer (ICT); (b) chemical structure of $\mathbf{o - C z}$, the red arrow represents space charge transfer (SCT); (c) molecular orbital amplitude plots of HOMO, LUMO, and $\mathrm{LUMO}+3$ of $\mathrm{o}-\mathrm{Cz}$, the red circle indicates the spatial orbital overlap of the donor and acceptor units; (d) photographs of $\mathrm{o}-\mathrm{Cz}$ under UV-light and NIR-light irradiation and after the excitation source is turned off.

In this contribution, we present the design and synthesis of a single-component organic molecule that exhibits TPUOP properties by dual-channel triplet harvesting. Based on $\boldsymbol{p}-\mathbf{C z}$ (Fig. 1a), which is a known UOP emitter, ${ }^{\mathbf{1 1}, \mathbf{1 2}}$ the more non-linear structural isomer $\boldsymbol{o}$-Cz that incorporates its donor (D) and acceptor (A) units with an ortho-connectivity, exhibits a folded conformation with close spatial proximity of the D and A units (Fig. 1b). Consequently, intramolecular charge transfer (CT) can take place through space CT (SCT) and through bond CT (ICT) simultaneously, affording an enhanced CT character to increase the TPA ability. The SCT effect causes a reduction in the singlet $\left(\mathrm{S}_{1}\right)$-triplet $\left(\mathrm{T}_{1}\right)$ energy gap $\left(\Delta E_{\mathrm{ST}}\right)$, which results in a highly efficient dual-channel triplet harvesting $\left(\Phi_{\mathrm{T}}=16.6 \%\right)$ via intersystem crossing (ISC) for phosphorescence and reverse ISC (RISC) for ultralong thermally activated delayed fluorescence (TADF). In addition, intramolecular $\mathrm{D}-\mathrm{A}$ interactions confine the vibrations of the excited molecule that suppresses nonradiative pathways. Benefiting from the SCT feature, $\boldsymbol{o}-\mathbf{C z}$ exhibits a stronger CT character and effective triplet harvesting, and consequently we successfully obtained the first singlecomponent TPUOP molecule. $\boldsymbol{o}-\mathbf{C z}$ exhibits obvious ultralong emission for $4 \mathrm{~s}$ after removal of the NIR-light excitation source (Fig. 1d).

\section{Results and discussion}

Compound $\boldsymbol{o}-\mathbf{C z}$ was synthesized by a CuI catalyzed Ullmann cross-coupling reaction of carbazole and 2BrBP (Fig. S1 $\dagger$ ). A suspension of $2 \mathrm{BrBP}(1 \mathrm{~g}, 3.83 \mathrm{mmol})$, carbazole $(0.77 \mathrm{~g}$,
$4.60 \mathrm{~mol})$, CuI (0.1 g) and 1,10-phenanthroline monohydrate $(0.1 \mathrm{~g})$ in DMF $(10 \mathrm{~mL})$ was stirred at reflux $\left(150{ }^{\circ} \mathrm{C}\right)$ overnight under an argon atmosphere. The reaction mixture was cooled to room temperature and subsequently poured into water (100 $\mathrm{mL}$ ), and the resulting precipitate was filtered and dried. The crude product was purified by flash column chromatography on silica gel (DCM : hexane $=1: 2$ ) to afford a yellowish solid $\boldsymbol{o}-\mathbf{C z}$ (0.72 g, yield $54.1 \%)$.

Time-dependent density functional theory (TD-DFT) was firstly performed to investigate the electronic distributions for the constitutional isomers $\boldsymbol{p}-\mathbf{C z}$ and $\boldsymbol{o}-\mathbf{C z}$ (extracted from the corresponding single crystal X-ray analysis). As depicted in Fig. S2, $\uparrow$ both the highest occupied molecular orbitals (HOMOs) and lowest unoccupied molecular orbitals (LUMOs) are separated on the carbazole (donor) and benzophenone (acceptor) units, respectively. Interestingly, the LUMO+3 of $\boldsymbol{o}-\mathbf{C z}$ is formed by the through-space orbital overlap of the donor and acceptor units, indicating the existence of SCT (Fig. 1c). ${ }^{22}$ In this regard, SCT is mediated by the spatial proximity of the donor and acceptor units and results in a smaller $\Delta E_{\mathrm{ST}}$ value of $0.21 \mathrm{eV}$, compared to $\boldsymbol{p}$ - $\mathbf{C z}(0.40 \mathrm{eV}) .^{23}$ The reduced $\Delta E_{\mathrm{ST}}$ value suggests the prospects for rapid triplet population via ISC, which is favorable for generation of phosphorescence. Additionally, the reduced density gradient analysis reveals the significant intramolecular interactions in the folded $\boldsymbol{o}$-Cz (Fig. 2b and S3, $\dagger$ including the green region of $\mathrm{D}-\mathrm{A}$ attractions and the brown region for steric hindrance). These interactions in $\boldsymbol{o}-\mathbf{C z}$ can effectively restrict the excited-state molecular vibrations and thus suppress non-radiative pathways. ${ }^{24}$ In contrast, the interaction regions are negligible in the more linear $\boldsymbol{p}-\mathbf{C z}$ molecule (Fig. 2a). Moreover, the second hyperpolarizability values $(\gamma)$, which are directly proportional to the TPA cross section, are $411.90 \times 10^{-36}$ esu and $6022.53 \times 10^{-36}$ esu for $\boldsymbol{p}-\mathbf{C z}$ and $\boldsymbol{o}-\mathbf{C z}$, respectively, which indicates better TPA capacities of $\boldsymbol{o}-\mathbf{C z}$.

Detailed analysis of the photophysical properties of $\boldsymbol{o}$-Cz was carried out by UV-vis absorption, photoluminescence (PL), and temperature-dependent transient decay studies. The UV-vis absorption profiles of compounds $\boldsymbol{o}-\mathbf{C z}$ and $\boldsymbol{p}-\mathbf{C z}$ in dilute THF are similar (Fig. 3a). The main vibronic absorption bands ranging around $230-270 \mathrm{~nm}, 270-300 \mathrm{~nm}$, and $300-350 \mathrm{~nm}$ are due to the localized $\pi-\pi *$ transitions of the acceptor and donor moieties. ${ }^{11,12}$ The broad and weak absorptions around 350$400 \mathrm{~nm}$ are caused by the ICT of the two isomers. For $\boldsymbol{o}-\mathbf{C z}$, the onset of absorption is red-shifted to $420 \mathrm{~nm}$, which originates from the SCT with much lower oscillator strength. ${ }^{25}$ Meanwhile,

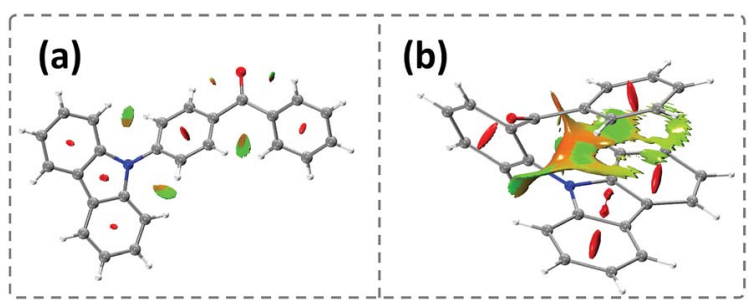

Fig. 2 Distribution of intramolecular interaction regions in (a) $p-C z$ and (b) o-Cz. 
(a)

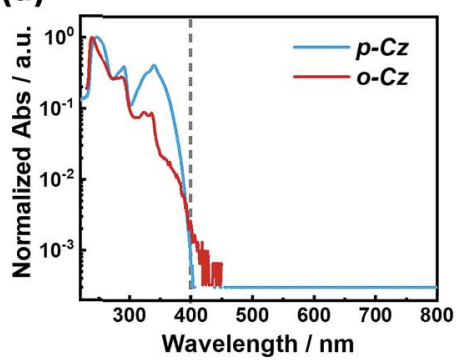

(d)

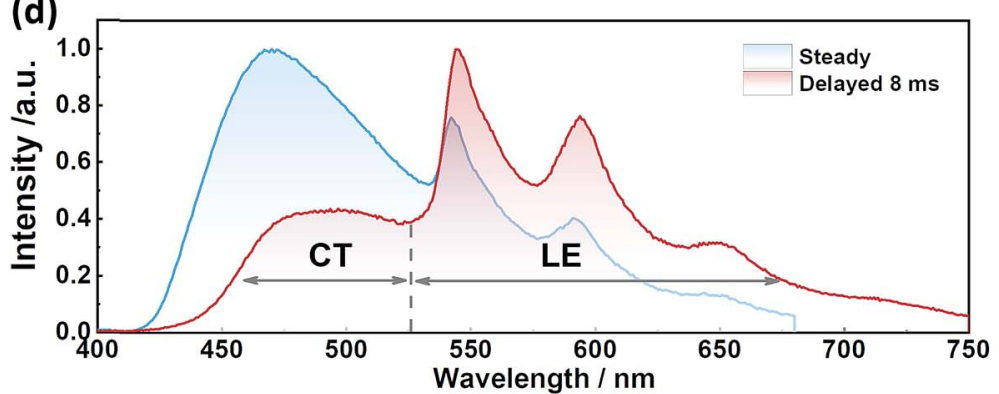

(f)

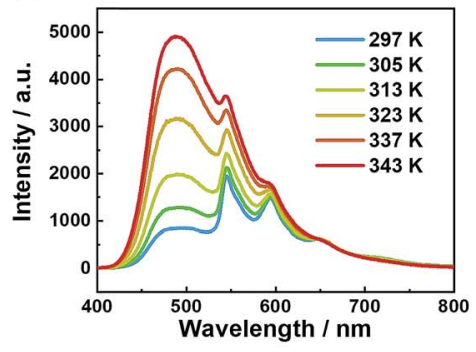

(b)

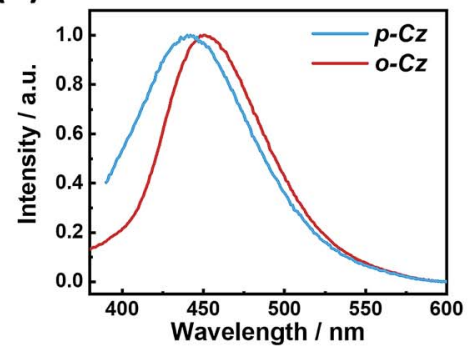

(g)

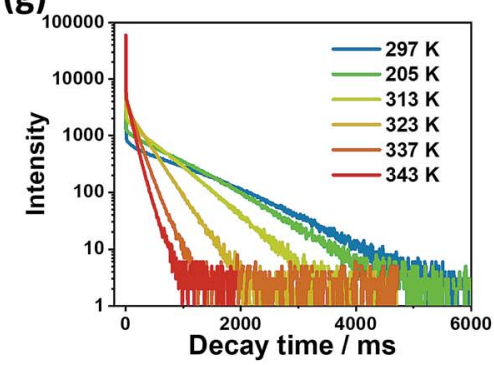

(c)

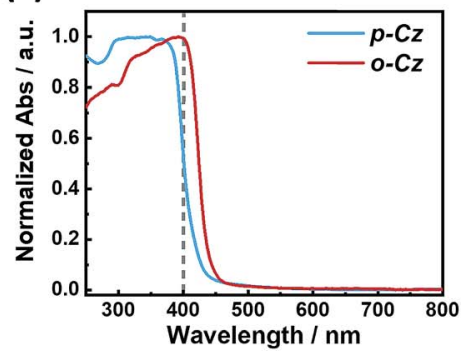

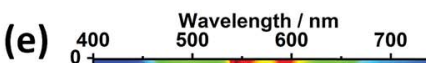

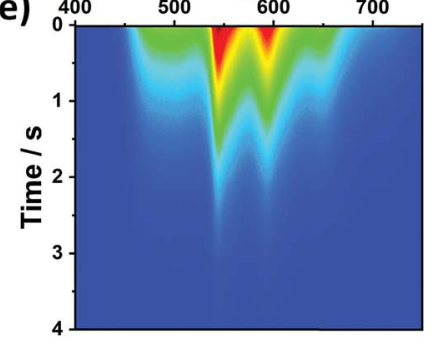

(h)

Fig. 3 Photophysical properties of $p-C z$ and $o-C z$ under ambient conditions. (a) UV-vis absorption spectra in THF solutions $\left(1 \times 10^{-5} \mathrm{M}\right)$, the grey dotted line at $400 \mathrm{~nm}$ represents the ultraviolet/visible region boundary; (b) PL spectra in THF solutions $\left(1 \times 10^{-5} \mathrm{M}\right)$; (c) UV-vis absorption spectra of crystalline powders; (d) steady-state and delayed $(8 \mathrm{~ms}) \mathrm{PL}$ spectra of $\mathrm{o}-\mathrm{Cz}$; (e) time-resolved emission spectra of $o-\mathrm{Cz}$ (excited at $365 \mathrm{~nm}$, integral time = $8 \mathrm{~ms}$ ); (f) temperature-dependent delayed emission; (g) lifetime decay curves of $o-\mathrm{Cz}$ (excited with $405 \mathrm{~nm}$, delay time = $8 \mathrm{~ms})$; and (h) plots of emission intensity (In /) versus temperature (1000/T) for o-Cz.

the emission maximum of $\boldsymbol{o}-\mathbf{C z}$ in solvents with different polarities exhibits a significant red-shift as the solvent polarity increases, from $424 \mathrm{~nm}$ in non-polar hexane to $492 \mathrm{~nm}$ in polar DMSO. This result demonstrates the ICT character of $\boldsymbol{o}-\mathbf{C z}$ (Fig. S4 $\dagger$ ). The PL emission of $\boldsymbol{o}-\mathbf{C z}$ in THF solution exhibits a bathochromic shift with a narrower full width at halfmaximum (FWHM) of $73 \mathrm{~nm}$ compared to that of the analogous $\boldsymbol{p}-\mathbf{C z}(90 \mathrm{~nm})$, indicating a stronger CT character with less excited-state structural vibrations in $\boldsymbol{o}$-Cz (Fig. $3 \mathrm{~b}$ ). ${ }^{15}$ In the solid state, the onset absorption of $\boldsymbol{o}-\mathbf{C z}$ is extended to the visiblelight region around $460 \mathrm{~nm}$, denoting further strengthening of the CT character in the aggregation state (Fig. 3c). Generally, the enhancement of the ICT effect is an effective strategy to increase the TPA ability. ${ }^{\mathbf{1 6}}$

The crystalline powder of $\boldsymbol{o}-\mathbf{C z}$ (Fig. S5†) exhibits an obvious UOP feature under ambient conditions after turning off the UV and NIR light excitation (Fig. 1d). Both the steady-state PL and delayed spectra of the crystalline $\boldsymbol{o}-\mathbf{C z}$ show two emission bands located around $472 \mathrm{~nm}$ (structureless with a CT character) and 522-700 $\mathrm{nm}$ (fine vibrations with a locally excited character) at room temperature (Fig. 3d). However, the two emission bands exhibit lifetimes of $0.79 \mathrm{~s}$ (472 nm) and $0.84 \mathrm{~s}$ (542 nm) (Fig. 3e), respectively, with totally different electronic configurations (CT and locally excited (LE) characters). We speculate that the different-emissive origins are responsible for the dual bands in $\boldsymbol{o}$-Cz. In a further experiment, the excitation-emission matrix spectra (Fig. S6 and S7a $\dagger$ ) show that the two emission bands can be excited from $260 \mathrm{~nm}$ to $450 \mathrm{~nm}$, with the strongest excitation at $428 \mathrm{~nm}$. The long-lived emissions of $\boldsymbol{o}-\mathbf{C z}$ can be triggered by both ultraviolet and visible light. The normalized steady-state PL spectra (Fig. S7b $\dagger$ ) with different excitation wavelengths are not overlapped around the LE zone (522-680 nm). Additionally, the delayed emission spectra (normalized at a delay time of $8 \mathrm{~ms}$ ) show distinct intensity around the CT zone (472 $\mathrm{nm})$ with UV (365 $\mathrm{nm}$ ) and visible light (405 $\mathrm{nm})$ excitation (Fig. S8†). These results indicate that the dual-channel of CT and LE long-lived emission originate from different emissive species in the crystalline $\boldsymbol{o}-\mathbf{C z}$. The emission intensities of the dual peaks in $\boldsymbol{o}-\mathbf{C z}$ show only a slight decrease in an oxygen atmosphere compared to that in a vacuum (Fig. S9†), which 
could be due to the crystalline morphology with relatively larger crystal dimensions than usual. However, the emission intensities of the dual peaks do show a slight decrease in an oxygen atmosphere compared to vacuum, indicating the involvement of triplet states in the overall luminescence of the molecule.

To gain detailed information regarding the photophysical properties of the dual bands with ultralong emission, the timeresolved PL of crystalline $\boldsymbol{o}-\mathbf{C z}$ was recorded at elevated temperature. In general, when the temperature increases the phosphorescence intensity should decrease dramatically due to thermal quenching. Abnormally, the delayed emission intensity of $\boldsymbol{o}$-Cz enhances as the temperature increases, especially for the CT bands, exhibiting linear plots of $\ln I$ (emission intensity) versus temperature (Fig. $3 \mathrm{f}$ and $\mathrm{h}$ ). Within this temperature range, no thermally induced phase transitions occur (Fig. S10†). The decay curves (collected at $472 \mathrm{~nm}$ ) of $\boldsymbol{o}$-Cz display prompt and delayed components (Fig. $3 \mathrm{~g}$ and $\mathrm{S} 11 \dagger$ ) and the proportions of the delayed component increase significantly with shortened lifetime as the temperature increases from $297 \mathrm{~K}$ to $343 \mathrm{~K}$. In addition, the intensity-dependent PL spectra (Fig. S12 and S13†) show a slope of 0.98 at low excitation density, which excludes the triplet-triplet annihilation process. These results are consistent with the TADF phenomenon, ${ }^{26-28}$ but show a much longer lifetime. With regard to TADF, the appropriate energy level distributions are essential for efficient RISC from the lowest triplet state to the singlet state. The $\Delta E_{\mathrm{ST}}$ values of $\boldsymbol{o}-\mathbf{C z}$ obtained in dilute THF solution, as well as the crystalline state (Fig. S14 and S15†), are $0.28 \mathrm{eV}$ and $0.17 \mathrm{eV}$, respectively, which are close to the theoretical calculations $(0.20 \mathrm{eV}$ and $0.21 \mathrm{eV}$, respectively). The obtained values are sufficient for thermal activation. Therefore, thermally-activated RISC from $T_{1}$ to $S_{1}$ is expected to harvest triplet excitons and results in the enhancement of the structureless CT band around $472 \mathrm{~nm}$ with a TADF character. The relatively large $\Delta E_{\mathrm{ST}}$ value of $0.61 \mathrm{eV}$ for $\boldsymbol{p}$ $\mathrm{Cz}$ (in dilute THF) means that RISC is less likely to occur (Table S3 $\dagger$ ). The reduced $\Delta E_{\mathrm{ST}}$ is ascribed to the SCT effect in $\boldsymbol{o}$-Cz. To date, the ultralong TADF materials have been found in zeolite and ionic crystals, in which the rigid matrices suppress the vibrations. ${ }^{29,30}$ Remarkably, the TADF lifetime of $\boldsymbol{o}-\mathbf{C z}$ is up to $0.79 \mathrm{~s}$, which to the best of our knowledge is the longest lifetime reported amongst the pure organic TADF emitters under ambient conditions. Such a long lifetime validates the unique intra- and intermolecular interactions that suppress the nonradiative pathways. This intriguing result reveals that the ultralong TADF phenomenon is also an alternative for realization of single component UOP materials. Furthermore, the ultralong emission of $\boldsymbol{o}-\mathbf{C z}$ exhibits temperature-sensitive features because of the enhancement of TADF emission at higher temperature (Fig. S16 and Table S4 $\dagger$ ). As revealed before, TADF molecules have great potential for two-photon lifetime imaging due to the significant ICT effect and two-component lifetime..$^{28}$

The long-lived LE emission of $\boldsymbol{o}$-Cz located around 522$700 \mathrm{~nm}$ shows red-shifted emission compared to the monomeric phosphorescence in dilute THF solution (Fig. S14b†), suggesting intermolecular electronic coupling within the crystalline lattice. ${ }^{11}$ Accordingly, single crystal X-ray diffraction analysis was performed to investigate the detailed molecular packing of $\boldsymbol{o}-\mathbf{C z}$ in the unit cell. $\boldsymbol{o}-\mathbf{C z}$ shows an ordered and condensed arrangement (Fig. S17 and S18†). The visual analyses of non-covalent interactions were carried out through an independent gradient model, ${ }^{31}$ which reveal the abundant interaction regions (green color) around adjacent molecules, as shown in Fig. S19. $\dagger$ Interestingly, the carbazole moieties stack tightly in a herringbone motif through the van der Waals interaction modes of $\mathrm{C}-\mathrm{H} \cdots \pi(2.894 \AA)$ (Fig. S19†), which are driven by the electrostatic interaction between the electron-deficient hydrogen atoms and the $\pi$-electron density. The herringbone packing modes prevent further molecular close-packing upon excitation and lead to predominantly $\mathrm{H}$-aggregate dimers. ${ }^{32,33} \mathrm{It}$ has been reported that carbazole-carbazole dimers in the solidstate can cause unwanted color variations in emission that could have negative implications for the device performance of TADF-OLEDs. ${ }^{34}$ The ortho-connectivity molecular design that is present in compound $\boldsymbol{o}-\mathbf{C z}$ should prevent these unwanted modes of dimerization and thereby be more desirable for TADFbased OLED devices with improved color purity. The H-dimers generate the intermolecular electronic coupling that can be verified by the TD-DFT result (Fig. S20 $\dagger$ ), affording the ultralong phosphorescence at $542 \mathrm{~nm}\left(\mathrm{~T}_{1}^{*}\right)$ with a LE-character. The two emission bands with the CT and LE character demonstrate the existence of dual-channel triplet harvesting pathways (TADF and $\mathrm{H}$-aggregation) in $\boldsymbol{o}-\mathbf{C z}$, which minimize the triplet concentration quenching and result in a high $\Phi_{\mathrm{T}}$ of $16.6 \%$. The dual-channel triplet harvesting shows a distinct advantage in the reduction of long-lived triplet quenching since the exciton concentration is very high with two-photon-excitation upon NIR laser irradiation.

Furthermore, the natural transition orbital (NTO) simulations and spin-orbital coupling (SOC) calculations of the excited states on both $\boldsymbol{o}$-Cz (Fig. S21†) and $\boldsymbol{p}$-Cz (Fig. S22 †) were carried out to evaluate the (R)ISC channels, which play pivotal roles in TADF and phosphorescence materials. As illustrated in Fig. 4b, the two excited triplet states $\left(T_{1}\right.$ and $\left.T_{2}\right)$ are located below the singlet state $\left(\mathrm{S}_{1}\right)$ in the folded molecule $\boldsymbol{o}$-Cz with narrow gaps of $0.04 \mathrm{eV}\left(\mathrm{S}_{1}\right.$ to $\left.\mathrm{T}_{1}\right)$ and $0.21 \mathrm{eV}\left(\mathrm{S}_{1}\right.$ to $\left.\mathrm{T}_{2}\right)$. In addition, the $\mathrm{S}_{1}$ state exhibits pure $\pi-\pi^{*}$ configuration, while the $\mathrm{T}_{1}$ and $\mathrm{T}_{2}$ consist of

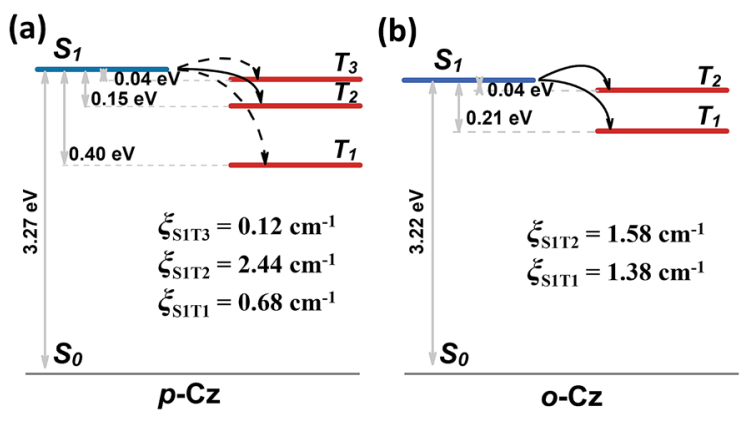

Fig. 4 Theoretically-calculated energy levels and spin-orbit coupling constants between $S_{1}$ and lower-lying $T_{n}$ with the corresponding crystal geometries of (a) $p-C z$ and (b) $o-C z$. Solid black arrows represent major channels with $\xi$ over $1 \mathrm{~cm}^{-1}$, and dashed black arrows represent minor channels with $\xi$ less than $1 \mathrm{~cm}^{-1}$. 
(a)

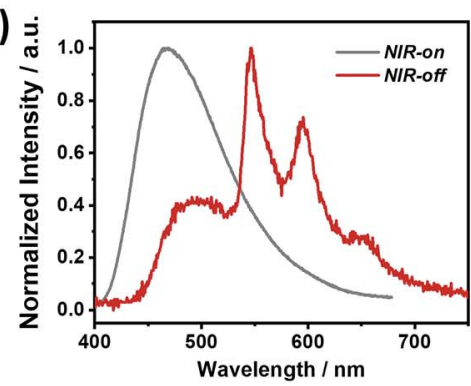

(b)

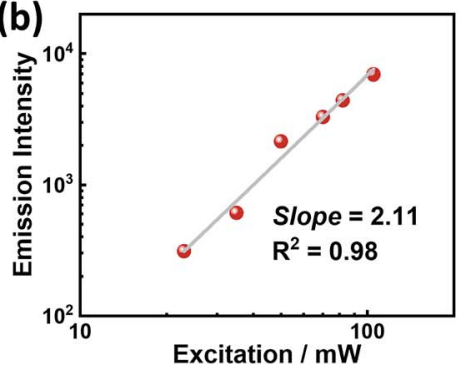

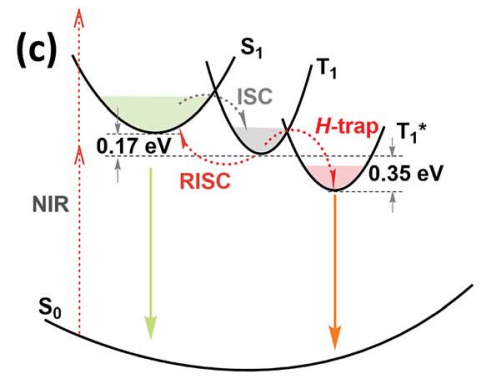

Fig. 5 (a) Two-photon-induced steady-state emission and delayed emission (delay $=8 \mathrm{~ms}$ ) of o-Cz excited with a $808 \mathrm{~nm}$ femtosecond laser; (b) plots of emission intensity varying with input laser density (collected at $542 \mathrm{~nm}$ ); (c) proposed mechanism of the two-photon induced ultralong phosphorescence in $\mathbf{o - C z}$. The energy levels are obtained with experimental spectra of the crystalline $\mathbf{o - C z}$.

hybrid $\mathrm{n}-\pi^{*}$ and $\pi-\pi^{*}$ configurations (Fig. S21 $\dagger$ ). According to El-Sayed's rule, the ISC is favored in $\boldsymbol{o}$-Cz between the $\mathrm{S}_{1}$ and the lower-lying triplet states $\left(\mathrm{T}_{1}\right.$ and $\left.\mathrm{T}_{2}\right)$. The SOC constants $(\xi)$ of the corresponding matrix elements in $\boldsymbol{o}$ - $\mathbf{C z}$ are $\xi\left(\mathrm{S}_{1}, \mathrm{~T}_{2}\right)=$ $1.58 \mathrm{~cm}^{-1}$ and $\xi\left(\mathrm{S}_{1}, \mathrm{~T}_{1}\right)=1.38 \mathrm{~cm}^{-1}$ (Fig. $4 \mathrm{~b}$ ). These values are relatively large for pure organic aromatic molecules and validate the efficient ISC channels. ${ }^{35}$ Additionally, RISC is facilitated by a narrow $\Delta E_{\mathrm{ST}}$ and large $\xi\left(\mathrm{S}_{1}, \mathrm{~T}_{1}\right)$ in $\boldsymbol{o}$ - $\mathbf{C z}$, attributed to the TADF process. Evidently, the theoretical calculations are consistent with the experimental results, which certifies the presence of dual-channel triplet harvesting in $\boldsymbol{o}-\mathbf{C z}$. In the more linear $\boldsymbol{p}-\mathbf{C z}$ (Fig. 4a), RISC is more difficult to take place due to a larger $\Delta E_{\mathrm{ST}}$ $(0.40 \mathrm{eV})$ and a smaller $\xi\left(\mathrm{S}_{1}, \mathrm{~T}_{1}\right)\left(0.68 \mathrm{~cm}^{-1}\right)$, preventing the TADF process.

Benefiting from the SCT feature, $\boldsymbol{o}$-Cz possesses a strong CT character and high emission quantum efficiency, which are prerequisites for designing efficient TPA molecules. ${ }^{16}$ In a further set of experiments, the PL intensity increases rapidly along with the enhancement of input NIR $(808 \mathrm{~nm})$ laser power (Fig. S23 $\dagger$ ). The quadratic dependence of the input power intensity verifies the two-photon-excited emission in $\boldsymbol{o}-\mathbf{C z}$ (Fig. 5b and S24†). Meanwhile, long-lived emission can be observed easily by the naked eye for several seconds after turning off the NIR laser (Fig. 1d). These results intensely demonstrate that $\boldsymbol{o}-\mathbf{C z}$ could indeed be a TPUOP molecule. The two-photon-excited delayed spectrum profile is overlapped with the single-photon induced emission (405 nm LED as the excitation source, $\approx 808 / 2 \mathrm{~nm}$, Fig. S25 $†$ ) including both CT and LE bands, which indicates the analogous excited state decay process after the single-photon or two-photon excitation in $\boldsymbol{o}$ Cz. Therefore, following simultaneous absorption of two NIR photons, triplet excitons can be generated through ISC from the lowest singlet state in the molecule (Fig. 5c). At this stage, partial triplet excitons undergo thermal up-conversion (RISC) that contributes to the $\mathrm{CT}$ emission with TADF characteristics. The remaining triplet excitons are "trapped" by the $\mathrm{H}$ aggregation $\left(\mathrm{T}_{1}^{*}\right)$ in the crystal lattice that results in the longlived LE-state emission. The triplet excitons are harvested in a dual-channel (TADF and "H-trap") to reduce the concentration quenching of the long-lived excitons. Due to the intra- and intermolecular interactions in the crystal lattice, $\boldsymbol{o}$-Cz exhibits ultralong emission due to the suppression of non-radiative pathways. In contrast, the more linear molecule $\boldsymbol{p}$ - $\mathbf{C z}$ exhibits a much lower phosphorescence efficiency $\left(\Phi_{\mathrm{T}}=0.3 \%\right)$ and vibronic fluorescence which indicates a decreased CT effect in the crystalline state (Fig. S27†), resulting in the faint emission after the NIR laser irradiation (Fig. S28†).

\section{Conclusions}

In conclusion, we designed and synthesized a spatially folded D-A type TPUOP molecule $\boldsymbol{o}-\mathbf{C z}$, which exhibits SCT features and can be excited by UV, visible, and NIR light. The enhanced CT character increases the TPA ability and the reduced $\Delta E_{\mathrm{ST}}$ facilitates both ISC and RISC processes to increase the triplet harvesting in a dual-channel. Meanwhile, the non-radiative pathways are suppressed due to the synergy of intra- and intermolecular interactions. These features of $\boldsymbol{o}$-Cz contribute to an ultralong lifetime of $0.84 \mathrm{~s}$ and a high $\Phi_{\mathrm{T}}$ of $16.6 \%$, which is among the best reported for heavy-atom-free UOP materials. Most importantly, we demonstrate that the $\boldsymbol{o}-\mathbf{C z}$ is an appropriate TPUOP molecule due to the enhanced CT character and efficient triplet harvesting. To the best of our knowledge, $\boldsymbol{o}-\mathbf{C z}$ is the first example of a single component TPUOP molecule. This molecular architecture may provide a new platform for designing more TPUOP molecules to broaden their applications.

\section{Conflicts of interest}

There are no conflicts to declare.

\section{Acknowledgements}

This work was financially supported by the National Natural Science Foundation of China (NSFC: 51803242, 51733010, 61605253 and 21672267), the Science and Technology Planning Project of Guangdong (2015B090913003), the China Postdoctoral Science Foundation (2017M620395) and the Fundamental Research Funds for the Central Universities. We appreciate Dr Shuang Li for help with the measurement of twophoton induced emission spectra and Dr Bingjia Xu for the further understanding of the results. 


\section{Notes and references}

1 S. Xu, R. Chen, C. Zheng and W. Huang, Adv. Mater., 2016, 28, 9920-9940.

2 Y. Xie, Y. Ge, Q. Peng, C. Li, Q. Li and Z. Li, Adv. Mater., 2017, 29, 1606829.

3 X. Zhen, Y. Tao, Z. An, P. Chen, C. Xu, R. Chen, W. Huang and K. Pu, Adv. Mater., 2017, 29, 1606665.

4 S. Fateminia, Z. Mao, S. Xu, Z. Yang, Z. Chi and B. Liu, Angew. Chem., Int. Ed., 2017, 56, 12160-12164.

5 J. Yang, X. Zhen, B. Wang, X. Gao, Z. Ren, J. Wang, Y. Xie, J. Li, Q. Peng, K. Pu and Z. Li, Nat. Commun., 2018, 9, 840.

6 Z. An, C. Zheng, Y. Tao, R. Chen, H. Shi, T. Chen, Z. Wang, H. Li, R. Deng, X. Liu and W. Huang, Nat. Mater., 2015, 14, 685-690.

7 W. Zhao, Z. He, J. W. Y. Lam, Q. Peng, H. Ma, Z. Shuai, G. Bai, J. Hao and B. Z. Tang, Chem, 2016, 1, 592-602.

8 D. Li, F. Lu, J. Wang, W. Hu, X.-M. Cao, X. Ma and H. Tian, J. Am. Chem. Soc., 2018, 140, 1916-1923.

9 S. Hirata, J. Phys. Chem. Lett., 2018, 9, 4251-4259.

10 R. Kabe and C. Adachi, Nature, 2017, 550, 384-387.

11 Z. Yang, Z. Mao, X. Zhang, D. Ou, Y. Mu, Y. Zhang, C. Zhao, S. Liu, Z. Chi, J. Xu, Y. Wu, P. Lu, A. Lien and M. Bryce, Angew. Chem., Int. Ed., 2016, 55, 2181-2185.

12 Y. Gong, G. Chen, Q. Peng, W. Z. Yuan, Y. Xie, S. Li, Y. Zhang and B. Z. Tang, Adv. Mater., 2015, 27, 6195-6201.

13 L. Bian, H. Shi, X. Wang, K. Ling, H. Ma, M. Li, Z. Cheng, C. Ma, S. Cai, Q. Wu, N. Gan, X. Xu, Z. An and W. Huang, J. Am. Chem. Soc., 2018, 140, 10734-10739.

14 S. Cai, H. Shi, J. Li, L. Gu, Y. Ni, Z. Cheng, S. Wang, W. Xiong, L. Li, Z. An and W. Huang, Adv. Mater., 2017, 29, 1701244.

15 Z. Mao, Z. Yang, Z. Fan, E. Ubba, W. Li, Y. Li, J. Zhao, Z. Yang, M. P. Aldred and Z. Chi, Chem. Sci., 2019, 10, 179-184.

16 H. M. Kim and B. R. Cho, Chem. Rev., 2015, 115, 5014-5055. 17 W. Denk, J. H. Strickler and W. W. Webb, Science, 1990, 248, 73-76.

18 J. Zhou, Q. Liu, W. Feng, Y. Sun and F. Li, Chem. Rev., 2015, 115, 395-465.

19 Q. Miao, C. Xie, X. Zhen, Y. Lyu, H. Duan, X. Liu, J. V. Jokerst and K. Pu, Nat. Biotechnol., 2017, 35, 1102-1110.
20 R. Gao, X. Mei, D. Yan, R. Liang and M. Wei, Nat. Commun., 2018, 9, 2798.

21 Z. Wang, C. Zhu, S. Yin, Z. Wei, J. Zhang, Y. Fan, J. Jiang, M. Pan and C. Su, Angew. Chem., Int. Ed., 2019, 58, 34813485.

22 L. Chen, Y. Wang, B. He, H. Nie, R. Hu, F. Huang, A. Qin, X. Zhou, Z. Zhao and B. Z. Tang, Angew. Chem., Int. Ed., 2015, 54, 4231-4235.

23 X. Chen, J. Jia, R. Yu, J. Liao, M. Yang and C. Lu, Angew. Chem., Int. Ed., 2017, 56, 15006-15009.

24 P. Rajamalli, N. Senthilkumar, P. Gandeepan, P. Huang, M. Huang, C. Ren-Wu, C. Yang, M. Chiu, L. Chu, H. Lin and C. Cheng, J. Am. Chem. Soc., 2016, 138, 628-634.

25 H. Tsujimoto, D. Ha, G. Markopoulos, H. S. Chae, M. A. Baldo and T. M. Swager, J. Am. Chem. Soc., 2017, 139, 4894-4900.

26 Z. Yang, Z. Mao, Z. Xie, Y. Zhang, S. Liu, J. Zhao, J. Xu, Z. Chi and M. P. Aldred, Chem. Soc. Rev., 2017, 46, 915-1016.

27 H. Uoyama, K. Goushi, K. Shizu, H. Nomura and C. Adachi, Nature, 2012, 492, 234-238.

28 W. Hu, L. Guo, L. Bai, X. Miao, Y. Ni, Q. Wang, H. Zhao, M. Xie, L. Li, X. Lu, W. Huang and Q. Fan, Adv. Healthcare Mater., 2018, 7, 1800299.

29 J. Liu, N. Wang, Y. Yu, Y. Yan, H. Zhang, J. Li and J. Yu, Sci. Adv., 2017, 3, e1603171.

30 B. Zhou and D. Yan, Adv. Funct. Mater., 2019, 29, 1807599.

31 T. Lu and F. Chen, J. Comput. Chem., 2012, 33, 580-592.

32 A. Kohler and H. Bassler, Electronic Processes in Organic Semiconductors, An Introduction, John Wiley \& Sons, 2015.

33 D. A. da Silva Filho, E.-G. Kim and J.-L. Brédas, Adv. Mater., 2005, 17, 1072-1076.

34 M. K. Etherington, N. A. Kukhta, H. F. Higginbotham, A. Danos, A. N. Bismillah, D. R. Graves, P. R. McGonigal, N. Haase, A. Morherr, A. S. Batsanov, C. Pflumm, V. Bhalla, M. R. Bryce and A. P. Monkman, J. Phys. Chem. C, 2019, 123, 11109-11117.

35 S. Cai, H. Shi, D. Tian, H. Ma, Z. Cheng, Q. Wu, M. Gu, L. Huang, Z. An, Q. Peng and W. Huang, Adv. Funct. Mater., 2018, 28, 1705045. 